\title{
Immunotherapy in patients with autoimmune disease
}

\author{
Sagar Rakshit, Julian R. Molina
}

Division of Medical Oncology, Mayo Clinic, Rochester, MN, USA

Contributions: (I) Conception and design: All authors; (II) Administrative support: None; (III) Provision of study materials or patients: None; (IV) Collection and assembly of data: All authors; (V) Data analysis and interpretation: All authors; (VI) Manuscript writing: All authors; (VII) Final approval of manuscript: All authors.

Correspondence to: Julian R. Molina, MD, PhD. Professor of Oncology, Consultant, Division of Medical Oncology and Molecular Medicine, Mayo Clinic, 200 First Street SW, Rochester, MN 55905, USA. Email: molina.julian@mayo.edu.

\begin{abstract}
Immune checkpoint inhibitors (ICIs) such as cytotoxic T-lymphocyte-associated protein 4 (CTLA-4), programmed cell death protein 1 (PD-1) and programmed cell death protein ligand 1 (PD-L1) inhibitors are widely used for the treatment of multiple cancers. Seven of these agents are currently FDA approved in the US as first or second line options for solid tumors and hematologic malignancies. These agents work by downregulating pathways that suppress T-cell activation and thereby mounting an immune response to the tumor. In general, ICI are well tolerated with only mild to moderate toxicity. However, in some patients severe immune-related adverse events (irAEs) that mimic the presentation of autoimmune diseases (AID) may occur. It is believed that irAEs occur due to disruption of immunologic self-tolerance, a mechanism that also seems to explain AID. Patients with pre-existing AID are usually excluded from prospective clinical trials due to concerns for flares of the underline AID. There is limited retrospective evidence supporting the use of ICI in patients with some pre-existing AID. These patients have an increased risk of malignancy and there is an unmet need to study ICIs in this population. This manuscript intends to review the current available evidence for the safety and activity of ICIs in patients with pre-existing AID. We summarize the reported use of ICI in patients with pre-existing AID according to the primary tumor site and type of ICI used.
\end{abstract}

Keywords: Immune checkpoint inhibitors (ICIs); autoimmune disease (AID); non-small cell lung cancer (NSCLC); stage IV; pre-existing autoimmune disease

Submitted Jan 28, 2020. Accepted for publication Jun 30, 2020.

doi: $10.21037 /$ jtd-2019-cptn-10

View this article at: http://dx.doi.org/10.21037/jtd-2019-cptn-10

\section{Introduction}

Immune checkpoint inhibitors (ICIs) are part of the armamentarium used for the treatment of several cancers and have impacted survival for patients with lung cancer, melanoma and other tumors. Ipilimumab [anti-cytotoxic T-lymphocyte-associated protein 4 (CTLA-4) monoclonal antibody] was the first FDA approved ICI in 2011. Since then, six more (ICIs) have been approved for anti-cancer therapy. In addition to ipilimumab, the programmed cell death protein 1 (PD-1) inhibitors pembrolizumab, nivolumab, cemiplimab and the programmed cell death protein ligand 1 (PD-L1) inhibitors atezolizumab, durvalumab, avelumab, were also granted approval. Approval for these PD-1/PD-L1 inhibitors includes melanoma, nonsmall cell lung cancer (NSCLC), small cell lung cancer, head and neck cancer, Merkel cell carcinoma, hepatocellular carcinoma, renal cell carcinoma, cervical cancer, triple negative breast cancer, gastric and gastroesophageal junction carcinoma, Hodgkin's lymphoma, urothelial cancer and microsatellite unstable tumors. The immune-related adverse events (irAEs) associated with the use of ICI likely result from disruption of the self-tolerance mechanisms and are higher for CTLA-4 inhibitors compared to PD-1/PDLlinhibitors $(1,2)$. Among the factors that increase the risk for irAEs, a personal history of autoimmune disease (AID) 
seems to be the most important one (1-4). Patients with pre-existing AIDs are usually excluded from prospective ICI clinical trials. Therefore, evidence regarding the safety and activity of ICI for this patient population is limited to retrospective studies.

\section{Mechanism of action}

The immune checkpoint pathway is in charge of regulating immune response and place a significant role in selftolerance and tumor immunology. Antigen non-specific signals work through co-stimulatory or co-inhibitory receptors on the $T$ cells (1). Co-stimulatory receptors such as CD28 increase $\mathrm{T}$ cell response, whereas co-inhibitory receptors such as CTLA-4 and PD- 1 attenuate $T$ cell response. CTLA-4 and PD-1 play a critical role in tolerance to self-antigens by guarding against autoimmune responses. For example, CTLA-4 regulates signals through the costimulatory receptor CD28 either by depleting signaling or by regulating endocytosis of CD80 and CD86 from antigen-presenting cells (APCs) (5). Likewise, PD-1 binds to the PD-1 ligand (PD-L1; also known as $\mathrm{B} 7-\mathrm{H} 1$ ) and PD-L2 (B7-H2) and inhibits not only tumor cell apoptosis, but also conversion of $\mathrm{T}$ effector cells to regulatory T-cells (Treg cells) and peripheral $\mathrm{T}$ effector cell exhaustion (6). Infiltration of tumors by $\mathrm{T}$ cells stimulates production of gamma-interferon which induces PD-L1 in the tumor microenvironment. PD-L1 then engages its receptor in the T-cells, PD-1, resulting in inhibition of T cells, allowing cancer cells to escape from the immune surveillance (7).

Failure of self-tolerance is one of the fundamental abnormalities in AIDs. In T cell-dependent inflammatory AIDs, there is an imbalance between effector $\mathrm{T}$ cells and functional Treg cells. In addition to auto-reactive $\mathrm{T}$ cells, several AIDs such as Graves' disease, myasthenia gravis, and systemic lupus erythematous (SLE) have autoreactive antibodies. Multiple studies have demonstrated a link between PD-1 dysregulation and autoimmunity (8). PD-1 deficient mice show a lupus-like phenotype and single-nucleotide polymorphisms on human PD-1 gene were associated with the development of lupus nephritis, type I diabetes and progressive multiple sclerosis (8). Polymorphism in the CTLA-4 gene have been link to ulcerative colitis risk in Asian populations (9). In addition, the toxicity profile for ICI resembles some of the manifestations of AIDs indicating a common mechanism between autoimmunity and irAEs seen with ICI (4).

\section{CTLA-4 inhibitors in patients with pre-existing AIDs}

Ipilimumab, a fully human monoclonal IgG1 antibody targeting CTLA-4, was the first ICI successfully tested for the treatment of metastatic melanoma. For patients previously treated, ipilimumab demonstrated in randomized trials robust and durable response that led to the FDA approval for the treatment of malignant melanoma in 2011 (10).

Two retrospective studies have evaluated the safety and clinical activity of ipilimumab in patients with pre-existing AIDs. The first of these studies done in 30 patients with metastatic malignant melanoma and pre-existing AIDs showed a $27 \%$ risk of developing a flare of the autoimmune condition requiring treatment with corticosteroids or infliximab (11). For this study, the median age was 59.5 years with $57 \%$ of patients being males. The median duration of the diagnosis of autoimmune disorder prior to initiation of ipilimumab was 13.5 years with $73 \%$ of patients having previously received immune modulators as part of their treatments (11). At the time of ipilimumab treatment $43 \%$ of patients had active AID requiring systemic treatment. Patients in this study had different pre-existing autoimmune disorders including 6 patients with rheumatoid arthritis, 6 with inflammatory bowel disease (IBD), 5 with psoriasis, 2 with multiple sclerosis, 2 with autoimmune thyroiditis (AIT) and 7 with other conditions. Toxicity associated with ipilimumab (33\%) was comparable to what has been described for patients with no autoimmune conditions and $50 \%$ of patients had no exacerbation of the underlying autoimmune disorder. Main immune related adverse events included thyroiditis, colitis, acute glaucoma and inflammatory arthritis. Response rate in the form of partial (PR) or complete (CR) response to ipilimumab was $20 \%$ with some responses lasting up to 9 months. The median progression-free survival (PFS) was 3.0 months (95\% CI, 2.0-8.3) and the median overall survival (OS) was 12.5 months (Table 1) (11).

Lee $e t$ al. reported the outcomes of eight patients with metastatic melanoma and rheumatoid arthritis treated with ipilimumab (12). The median age was 72 years with $50 \%$ of patients reporting prior use of diseasemodifying anti-rheumatic drugs (DMARD) including methotrexate and etanercept. Five out of 8 patients $(62.5 \%)$ discontinued ipilimumab due to irAEs. Four had grade 1 and 2 irAEs. One patient had grade 3 flare of arthritis that responded well to glucocorticoids and non-steroidal anti- 
Table 1 Retrospective studies using CTLA-4 inhibitors in patients with pre-existing AID

\begin{tabular}{lccccc}
\hline ICl & Patients & Tumor & G3-4 irAEs & ORR & Author \\
\hline Ipilimumab & 29 & Melanoma & $24 \%$ & $31 \%$ & Johnson (11) \\
Ipilimumab & 8 & Melanoma & $25 \%$ & $50 \%$ & Lee (12) \\
\hline
\end{tabular}

CTLA-4, cytotoxic T-lymphocyte-associated protein 4; irAEs, immune-related adverse events; AID, autoimmune disease; ICl, immune checkpoint inhibitor.

Table 2 Retrospective studies using PD-1/PD-L1 inhibitors in patients with pre-existing AID

\begin{tabular}{|c|c|c|c|c|c|}
\hline $\mathrm{ICl}$ & Patients & Tumor types & G3-4 irAEs & ORR & Author \\
\hline PD-1/PD-L1 & 19 & Melanoma & $16 \%$ & $32 \%$ & Gutzmer (18) \\
\hline PD-1/PD-L1 & 56 & NSCLC & $38 \%$ & $22 \%$ & Leonardi (19) \\
\hline CTLA-4, PD-1, PD-L1 & 16 & Melanoma, NSCLC, other malignancies & $38 \%$ & Not available & Ritchter (20) \\
\hline CTLA-4, PD-1, PD-L1 & 112 & Melanoma, NSCLC, other malignancies & $38 \%$ & $48-54 \%$ & Tison (22) \\
\hline CTLA-4, PD-1, PD-L1 & 102 & Melanoma, NSCLC, other malignancies & $41 \%$ & $48 \%$ & Abu-Sbeih (23) \\
\hline PD-1/PD-L1 & 85 & Renal cell carcinoma, urothelial cancer & $9.4 \%$ & $38.1 \%$ & Cortellini (24) \\
\hline
\end{tabular}

PD-1, programmed cell death protein 1; PD-L1, programmed cell death protein ligand 1; AID, autoimmune disease; ICI, immune checkpoint inhibitor; CTLA-4, cytotoxic T-lymphocyte-associated protein 4; NSCLC, non-small cell lung cancer; ORR, overall response rate; irAEs, immune-related adverse events.

inflammatory drugs. One patient required infliximab and surgical intervention. Response rate in the form of PR was seen in $57 \%$ of patients with $37 \%$ having stable disease (Table 1) (12).

\section{PD-1/PD-L1 inhibitors in patients with pre- existing AIDs}

The PD-1 was discovered while trying to identify a hematopoietic progenitor cell line that undergoes programmed cell death upon IL-3 deprivation (13). Soon after, using a PD-1-Ig fusion protein, the ligand, PD-L1 was discovered (14). PD-L1 is expressed within the tumor microenvironment and upregulated on many solid tumors (15). Blockade of the interaction between PD-1 and PD-L1 with ICI results in antitumor activity mediated by T-cells (16). Six PD-1/PD-L1 inhibitors have demonstrated clinical activity and are currently FDA-approved for the treatment of several cancers. There are no prospective studies evaluating the safety and clinical activity of PD-1/PD-L1 inhibitors in patients with pre-existing AIDs. However, we have data obtained from several retrospective analyses suggesting that is safe and effective to use ICI in patients with pre-existing AID. We will discuss next the data from several of these studies (Table 2).

A nationwide multicenter retrospective study from France evaluated 112 patients treated with ICI and preexisting AID (22). Main cancer types were melanoma $(\mathrm{n}=66,58.9 \%)$ and NSCLC $(\mathrm{n}=40 ; 35.7 \%)$. Median age for the overall population was 66.5 years with $57 \%$ of cases being males. The pre-existing AID included 31 patients with psoriasis, 20 with rheumatoid arthritis, 14 with IBD, 7 with SLE, 7 with polymyalgia rheumatica, 5 with spondyloarthritis and 28 with other conditions. The median duration of the pre-existing autoimmune disorder was 20 years with $30 \%$ of patients having received previous immunosuppressive therapy (22). Only $3.2 \%$ of cases were on active immunosuppressive therapy at the time of initiation of the ICI. The study showed that flares from a pre-existing autoimmune condition were common $(n=47 ; 42 \%)$. Disease flares were severe in $30.5 \%$ of cases (CTCAE grades 3 and 4) requiring treatment with steroids in 28 of 52 patients (54\%) (22). Six patients (21\%) received methotrexate or azathioprine, and 3 patients $(11 \%)$ received 
a biologic DMARD [tumor necrosis factor (TNF) inhibitor for IBD flares]. Forty-seven patients (42\%) experienced irAE(s) not related to their pre-existing AID. Death due to immune-related toxicity was seen in $3.2 \%$ (22). The most frequent irAEs were colitis, thyroiditis, vitiligo and hypophysitis. Ipilimumab-related immune toxicities were more severe than PD-1/PD-L1 inhibitor-related toxicities (55\% vs. 38\%). Interestingly, PFS and OS were longer in patients that developed irAEs or a flare of the AID. This study suggested that ICIs in patients with pre-existing autoimmune disorders may have a better efficacy [overall response rate (ORR) was 49\%, 32\% PR and 16\% CR] (22).

A retrospective multicenter study initiated by the German Dermatologic Cooperative Oncology Group included 41 patients with metastatic melanoma (18). In this analysis, patients were divided into two groups, group A $(n=19)$ included patients with pre-existing AID prior to PD-1 inhibitor use and group B ( $\mathrm{n}=22)$ was limited to patients with ipilimumab-related autoimmune events (18). Pre-existing AID included psoriasis, vasculitis, rheumatoid arthritis, myositis, polymyalgia rheumatica, spondylarthritis, sarcoidosis, IBD, Guillain Barré, transverse myelitis, multiple sclerosis, myasthenia gravis, AIT and hypophysitis (18). For group A, the median age was 54 with $53 \%$ of patients being female. Nivolumab $(63 \%)$ and pembrolizumab (37\%) were the main ICI used. For group $\mathrm{B}$ (patients with ipilimumab-triggered irAE), the median age was 52 years (59\% males) (18). Nivolumab was given to $41 \%$ of cases and pembrolizumab to $59 \%$. For group A the frequency of fares on the AID was $40 \%$ compared to $4.5 \%$ for group B. For this study, fares were more common in patients with underlying rheumatologic disorders (18).

In a retrospective study of 16 patients treated at the Mayo Clinic with melanoma $(n=10)$, NSCLC $(n=4)$ and hematologic malignances $(n=2)$, found immune-related side effects in 6 of 16 patients treated with ICIs (20). The pre-existing autoimmune disorders included 5 patients with rheumatoid arthritis, 5 with polymyalgia rheumatic, 2 with Sjögren's syndrome and 2 with systemic lupus erythematosus. Ten patients $(63 \%)$ had previously received a DMARD, but only 2 patients were on active systemic treatment at the time of ICI initiation. The median age was 68.5 years ( $81 \%$ females). Immune related adverse events were seen in $38 \%$ of cases and required treatment with glucocorticoids and discontinuation of immune check inhibitors (20). In this small study, patients who experienced irAEs tended to survive longer than those who did not have an irAE (20).
A systematic review done on publications through 14 September 2017 identified 123 patients treated with ICI and pre-existing AID (25). The study analyzed 49 publications from MEDLINE, EMBASE, Web of Science, PubMed Epubs, and the Cochrane Central Register of Controlled Trials. Most publications were case reports [39] or case series [4] but there were 5 retrospective observational studies (25). The median age was 61.4 years (57\% male) and the tumor types included metastatic melanoma, NSCLC, renal cell carcinoma and Merkel cell carcinoma. Psoriasis was the most common pre-existing disorder (22.8\%) followed by rheumatoid arthritis (16.3\%), IBD (10.6\%), autoimmune thyroid disease $(8.9 \%)$, multiple sclerosis (4.1\%), myasthenia gravis $(3.3 \%)$ and other autoimmune disorders (34\%) (25). Exacerbation of the pre-existing AID was seen in $41 \%$ and $75 \%$ reported irAEs. Colitis and hypophysitis were the most commonly seen irAEs (14\% and $5 \%)$. Three patients had a renal transplant rejection that occurred at median of 8 days (range, 7 to 21 days). There was no significant difference in the occurrence of adverse events in patients with active $v s$. inactive pre-existing AID (67\% vs. $75 \%$ ) (25). Active disease was defined as the presence of ongoing symptoms. Adverse events required treatment with high-dose corticosteroids or other DMARD such as infliximab, methotrexate, hydroxychloroquine, azathioprine, adalimumab, secukinumab, apremilast, or rituximab or other forms of immunosuppressive therapies (glatiramer acetate or vedolizumab) were seen, in $62 \%$ of patients (25). Discontinuation of treatment due to adverse events happened in 21 patients $(17.1 \%)$ with 5 patients switching to another form of ICI. The study found no differences in frequency of adverse events in patients with active $v s$. inactive pre-existing AID, whereas those receiving immunosuppressive therapy at initiation of ICI therapy seemed to have fewer adverse events than those not receiving therapy (25).

Leonardi conducted a retrospective study in patients with NSCLC and a pre-existing diagnosis of AID treated with a PD-1/PD-L1 inhibitor between May 2015 and December 2017 (19). The qualifying AID for the study included rheumatologic $(45 \%)$, dermatologic $(29 \%)$, endocrine $(16 \%)$, gastrointestinal (GI) (11\%), neurologic (5\%), and autoimmune hemolytic anemia (5\%) (19). Ten (18\%) of 56 patients included in the analysis had active symptoms from their AID and 11 (20\%) were on immunosuppressant or immunomodulatory agents (19). Exacerbations of underlying AID occurred in $23 \%$ (87\% grade 1 or 2, $13 \%$ grade 2, no grades 3 and 4) (19). Flares occurred more 
often in patients with rheumatologic disorders compared with patients with non-rheumatologic AID (40\% vs. 10\%, respectively; $\mathrm{P}=0.01$ ) (19). No flares were seen in patients with IBD or neurologic AID. Treatment for the irAEs included supportive care and steroids (19). In total, $55 \%$ of patients developed either an AID flare or an irAE, and only three patients (5\% of the cohort) developed both an AID flare and a separate irAE. Response rates in the form of a PR was seen in 11 patients (22\%), SD in 15 patients (31\%) and progressive disease in 23 patients (47\%) (19).

Danlos conducted a retrospective comparison of toxicity, OS and the best ORR in patients treated with ICI and pre-existing AID vs. patients without AIDs (21). A total of 45 patients from the prospective REISAMIC registry (Registry of Severe Adverse Events of Immunomodulating Monoclonal Antibodies in Oncology) seen between June 2014, and December 2016 were included in the analysis and compared to 352 AID-free patients also from the REISAMIC registry over the same period (21). Thirty-seven (82.2\%) had a single pre-existing AID, and eight (17.8\%) had two concomitant AIDs. Thirty-six patients $(80.0 \%)$ had a diagnosis of melanoma, $6(13.3 \%)$ NSCLC and 3 had other cancers $(6.7 \%$; renal cancer, penile epidermoid cancer and Merkel cell carcinoma). The ICIs given to patients were in the either pembrolizumab, nivolumab or avelumab. A flare of the pre-existing AID was seen in $55 \%$ of patients and irAEs were seen in $44.4 \%$ (21). Patients received specific treatments for the irAEs and the ICI was maintained in $75 \%$ of cases. When compared to the 352 AID-free patients enrolled in REISAMIC over the same period of time, irAEs were more frequent in the group with AID (44.4\% vs. 29\%) (21). The ORR was 38\% (9\% with a CR and $29 \%$ with a PR) in AID patients and $28 \%$ (7\% with a CR and $21 \%$ with a PR) in AID-free patients. There was no difference in OS between AID and AID-free patients $(\mathrm{P}=0.38)(21)$.

A "real-world" retrospective, multicenter observational study performed by an Italian group reviewed consecutive patients with advanced cancer, treated with $\mathrm{PD}-1 / \mathrm{PD}-\mathrm{L} 1$ inhibitors and pre-existing AID (24). Among 751 patients enrolled, $85(11.3 \%)$ had a pre-existing AID. Clinically active pre-existing AID included psoriasis in 40\%, $40 \%$ rheumatologic (rheumatoid arthritis and polymyalgia rheumatic), 13.3\% GI (Crohn's disease) and 6.6\% multiple sites (scleroderma and AIT) (24). The median age was 69 years with $66.4 \%$ of patients being male. NSCLC was the predominant tumor $(65.5 \%)$ followed by melanoma (21.2\%), renal cell carcinoma (12.5\%) and other tumors
(0.8\%). Most patient received pembrolizumab (75.8\%) with nivolumab (24). For the group of patients with preexisting AIDs, grade 1 and 2 irAEs were seen in $65.9 \%$ and grades 3 and 4 irAEs in $9.4 \%$ with no statistical difference when compared to the group without pre-existing AID (8.8\%, $\mathrm{P}=0.8663)$ (24). The discontinuation rate after irAEs for patients with pre-existing AID was 7\%, whereas it was $7.2 \%$ for patients without pre-existing AID (24). The ORR among patients with pre-existing inactive AID was $38.1 \%$ (95\% CI: 24.4-56.6\%) and with pre-existing active AID was 50.0\% (95\% CI: $23.0-76.9 \%)$. For the group with pre-existing AID the ORR was $38.5 \%$ (95\% CI: 31.5-40.5\%) (24).

A multicenter, retrospective study of patients with documented IBD treated with ICI included 102 patients, 51 with Crohn's disease and 51 with ulcerative colitis (23). All patients needed to have clear documentation of underlying IBD either by histology or by being on active IBD-specific therapy. Eighty-five patients were treated with PD-1/PD-L1 inhibitors and 17 received treatment with a CTLA-4 inhibitor (23). Most patients had melanoma (44\%), followed by NSCLC (23\%), GI cancers (17\%) genitourinary cancers (7\%), head and neck cancers (4\%) and other cancers (6\%) (23). The median age was 65 years and $68 \%$ of the patients were males. Of the 102 patients, 42 (41\%) had a GI adverse event and 23 patients needed to stop ICI because of the GI adverse event (23). The overall rate of GI adverse event of any grade was significantly higher for patients without IBD (11\%) compared to patients with underlying IBD $(\mathrm{P}<0.001)(23)$. In addition, 4 patients developed colonic perforation (2 with Crohn's disease and 2 with ulcerative colitis). Stable disease or PR was seen in $48 \%$ of patients (23).

\section{Summary}

Although the evidence available for the use of ICI in patients with pre-existing AID is limited to retrospective analysis, for most AID, the use of ICI may not only be safe but also effective. Therefore, we recommend considering treatments with ICI for most patients with pre-existing AID including those with an active AID. Special considerations should be given to patients with neurologic AID, such as myasthenia gravis as the risk for a flare of the underlying condition may result in a life-threatening event. We also recommend for patients with other active or inactive AIDs to involve other specialists, particularly AID specialist. Additionally, it is important to have a good assessment regarding the activity of the pre-existing AID before 
initiation of an ICI. A multispecialty approach would also facilitate a coordinated management of potential irAEs.

Considering the limitations of retrospective studies and the numbers of patients with particular AID being small, it appears that a flare is more common in certain disorders. A flare seems to be more common in patients with rheumatological disorders (52\% of patients experiencing flares in the case series of Menzies et al.) and psoriasis (50\% in the series of Menzies et al.). In the Abu-Sbeih study done in patients with IBD, the rate of GI adverse events was high indicating that pre-existing IBD increases the risk of severe GI adverse events in patients treated with ICI (23). The risk of irAEs seems to be higher with Ipilimumab than with PD-1/PD-L1 inhibitors $(11,12)$. Patients receiving high levels of immunosuppression for AID control should also be approached with caution as these patients may be very difficult to manage if they develop irAEs and high doses of immunosuppression at the time of ICI initiation may compromise efficacy of ICI treatments.

\section{Conclusions}

Patients with pre-existing AID who could be considered for ICI treatment include those with a non-lifethreatening AID, non-neurologic AID and good control of the pre-existing AID. We recommend involvement of a multidisciplinary team in the decision to initiate ICI therapy. When possible, a targeted treatment of the preexisting AID should be considered to avoid higher doses of systemic immunosuppression.

\section{Acknowledgments}

Funding: This work was supported in part by a generous gift from The Whitney and Betty MacMillan Fund in Lung Cancer.

\section{Footnote}

Provenance and Peer Review: This article was commissioned by the Guest Editor (Chi Wan Koo) for the series "Contemporary Practice in Thoracic Neoplasm Diagnosis, Evaluation and Treatment" published in Fournal of Thoracic Disease. The article was sent for external peer review organized by the Guest Editor and the editorial office.

Conflicts of Interest: Both authors have completed the ICMJE uniform disclosure form (available at http://dx.doi. org/10.21037/jtd-2019-cptn-10). The series "Contemporary Practice in Thoracic Neoplasm Diagnosis, Evaluation and Treatment" was commissioned by the editorial office without any funding or sponsorship. The authors have no other conflicts of interest to declare.

Ethical Statement: Both authors are accountable for all aspects of the work in ensuring that questions related to the accuracy or integrity of any part of the work are appropriately investigated and resolved.

Open Access Statement: This is an Open Access article distributed in accordance with the Creative Commons Attribution-NonCommercial-NoDerivs 4.0 International License (CC BY-NC-ND 4.0), which permits the noncommercial replication and distribution of the article with the strict proviso that no changes or edits are made and the original work is properly cited (including links to both the formal publication through the relevant DOI and the license). See: https://creativecommons.org/licenses/by-nc-nd/4.0/.

\section{References}

1. Papaioannou NE, Beniata OV, Vitsos P, et al. Harnessing the immune system to improve cancer therapy. Ann Transl Med 2016;4:261.

2. Postow MA, Sidlow R, Hellmann MD. Immune-Related Adverse Events Associated with Immune Checkpoint Blockade. N Engl J Med 2018;378:158-68.

3. Kumar V, Chaudhary N, Garg M, et al. Current Diagnosis and Management of Immune Related Adverse Events (irAEs) Induced by Immune Checkpoint Inhibitor Therapy. Front Pharmacol 2017;8:49.

4. Vaddepally RK, Kharel P, Pandey R, et al. Review of Indications of FDA-Approved Immune Checkpoint Inhibitors per NCCN Guidelines with the Level of Evidence. Cancers (Basel) 2020;12:738.

5. Walunas TL, Lenschow DJ, Bakker CY, et al. CTLA-4 can function as a negative regulator of $\mathrm{T}$ cell activation. Immunity 1994;1:405-13.

6. Terawaki S, Chikuma S, Shibayama S, et al. IFN-alpha directly promotes programmed cell death-1 transcription and limits the duration of T cell-mediated immunity. J Immunol 2011;186:2772-9.

7. Pardoll DM. The blockade of immune checkpoints in cancer immunotherapy. Nat Rev Cancer 2012;12:252-64.

8. Nielsen C, Hansen D, Husby S, et al. Association of a putative regulatory polymorphism in the PD-1 gene 
with susceptibility to type 1 diabetes. Tissue Antigens 2003;62:492-7.

9. $\mathrm{Ng} \mathrm{SC}$, Tsoi KK, Kamm MA, et al. Genetics of inflammatory bowel disease in Asia: systematic review and meta-analysis. Inflamm Bowel Dis 2012;18:1164-76.

10. Hodi FS, O'Day SJ, McDermott DF, et al. Improved survival with ipilimumab in patients with metastatic melanoma. N Engl J Med 2010;363:711-23.

11. Johnson DB, Sullivan RJ, Ott PA, et al. Ipilimumab therapy in patients with advanced melanoma and preexisting autoimmune disorders. JAMA Oncol 2016;2:234-40.

12. Lee B, Wong A, Kee D, et al. The use of ipilimumab in patients with rheumatoid arthritis and metastatic melanoma. Ann Oncol 2016;27:1174-7.

13. Ishida $Y$, Agata $Y$, Shibahara K, et al. Induced expression of PD-1, a novel member of the immunoglobulin gene superfamily, upon programmed cell death. EMBO J 1992;11:3887-95.

14. Tashiro K, Tada H, Heilker R, et al. Signal sequence trap: a cloning strategy for secreted proteins and type I membrane proteins. Science 1993;261:600-3.

15. Dong H, Strome SE, Salomao DR, et al. Tumor-associated B7-H1 promotes T-cell apoptosis: a potential mechanism of immune evasion. Nat Med 2002;8:793-800.

16. Iwai Y, Ishida M, Tanaka Y, et al. Involvement of PD-L1 on tumor cells in the escape from host immune system and tumor immunotherapy by PD-L1 blockade. Proc Natl Acad Sci U S A 2002;99:12293-7.

17. Menzies AM, Johnson DB, Ramanujam S, et al. AntiPD-1 therapy in patients with advanced melanoma and preexisting autoimmune disorders or major toxicity with ipilimumab. Ann Oncol 2017;28:368-76.

18. Gutzmer R, Koop A, Meier F, et al. Programmed cell

Cite this article as: Rakshit S, Molina JR. Immunotherapy in patients with autoimmune disease. J Thorac Dis 2020;12(11):70327038. doi: 10.21037/jtd-2019-cptn-10 death protein-1 (PD-1) inhibitor therapy in patients with advanced melanoma and preexisting autoimmunity or ipilimumab-triggered autoimmunity. Eur J Cancer 2017;75:24-32.

19. Leonardi GC, Gainor JF, Altan M, et al. Safety of Programmed Death-1 Pathway Inhibitors Among Patients With Non-Small-Cell Lung Cancer and Preexisting Autoimmune Disorders. J Clin Oncol 2018;36:1905-12.

20. Richter MD, Pinkston O, Kottschade LA, et al. Brief Report: Cancer Immunotherapy in Patients with Preexisting Rheumatic Disease: The Mayo Clinic Experience. Arthritis Rheumatol 2018;70:356-60.

21. Danlos FX, Voisin AL, Dyevre V, et al. Safety and efficacy of anti-programmed death 1 antibodies in patients with cancer and pre-existing autoimmune or inflammatory disease. Eur J Cancer 2018;91:21-9.

22. Tison A, Quere G, Misery L, et al. Safety and efficacy of immune checkpoint inhibitors in patients with cancer and preexisting autoimmune disease: a nationwide, multicenter cohort study. Arthritis Rheumatol 2019;71:2100-11.

23. Abu-Sbeih H, Faleck DM, Ricciuti B, et al. Immune Checkpoint Inhibitor Therapy in Patients with Preexisting Inflammatory Bowel Disease. J Clin Oncol 2020;38:576-83.

24. Cortellini A, Buti S, Santini D, et al. Clinical Outcomes of Patients with Advanced Cancer and Pre-Existing Autoimmune Diseases Treated with Anti-Programmed Death-1 Immunotherapy: A Real-World Transverse Study. Oncologist 2019;24:e327-37.

25. Abdel-Wahab N, Shah M, Lopez-Olivo MA, et al. Use of Immune Checkpoint Inhibitors in the Treatment of Patients with Cancer and Preexisting Autoimmune Disease: A Systematic Review. Ann Intern Med 2018;168:121-30. 